\title{
Karyotype differentiation of four Cestrum species (Solanaceae) based on the physical mapping of repetitive DNA
}

\author{
Jéferson Nunes Fregonezi ${ }^{1}$, Thiago Fernandes ${ }^{1}$, José Marcelo Domingues Torezan ${ }^{2}$, Ana Odete S. Vieira ${ }^{2}$ \\ and André Luís Laforga Vanzela ${ }^{1}$ \\ ${ }^{1}$ Universidade Estadual de Londrina, Centro de Ciências Biológicas, Departamento de Biologia Geral, \\ Londrina, PR, Brazil. \\ ${ }^{2}$ Universidade Estadual de Londrina, Centro de Ciências Biológicas, Departamento de Biologia Animal \\ e Vegetal, Londrina, PR, Brazil.
}

\begin{abstract}
We studied the karyotypes of four Brazilian Cestrum species (C. amictum, $C$. intermedium, $C$. sendtnerianum and $C$. strigilatum) using conventional Feulgen staining, C-Giemsa and C-CMA ${ }_{3}$ DAPI banding, induction of cold-sensitive regions (CSRs) and fluorescent in situ hybridization (FISH) with rDNA probes. We found that the karyotypes of all four species was $2 n=2 x=16$, with, except for the eighth acrocentric pair, a predominance of meta- and submetacentric chromosomes and various heterochromatin classes. Heterochromatic types previously unreported in Cestrum as neutral C-CMA ${ }_{3}{ }^{\circ} \mathrm{DAPI}^{0}$ bands, $\mathrm{CMA}_{3}{ }^{+}$bands not associated with NORs, and C-Giemsa/CSR/DAPI bands were found. The heterochromatic blocks varied in size, number, position and composition. The 45S rDNA probe preferentially located in the terminal and subterminal regions of some chromosomes, while 5S rDNA appeared close to the centromere of the long arm of pair 8 . These results suggest that karyotype differentiation can occur mainly due to changes in repetitive DNA, with little modification in the general composition of the conventionally stained karyotype.
\end{abstract}

Key words: Cestrum, chromosome banding, CSRs, karyotype evolution, rDNA probes.

Received: August 24, 2004; Accepted: May 31, 2005.

\section{Introduction}

Along with Vestia (Willd) and Sessea (Ruiz et Pav) the genus Cestrum (L.) is part of the tribe Cestreae (G. Don) of the Cestroideae - Solanaceae). The genus Cestrum contains about 250 species distributed in the tropical and subtropical regions of the Americas (D'Arcy, 1986) and includes herbs, shrubs and trees up to $12 \mathrm{~m}$ in height. Members of this genus are recognized by their actinomorphic flowers with tubular-infundibuliforme corolla, five stamens attached to the corolla tube up to the middle or higher, bilocular ovary with berry-like fruits, few compressed seeds and a straight embryo (Cronquist, 1988). The karyotype of Cestrum species contains $2 \mathrm{n}=2 \mathrm{x}=16$ chromosomes of similar size and shape, most of which are meta- or submetacentric except for one subtelocentric pair (Berg and Greilhuber, 1992, 1993a,b). In general, Solanaceae species

Send correspondence to André L.L. Vanzela. Universidade Estadual de Londrina, Centro de Ciências Biológicas, Departamento de Biologia Geral, Laboratório de Biodiversidade e Restauração de Ecossistemas, Caixa Postal 6001, 86051-990 Londrina, PR, Brazil. E-mail: andrevanzela@uel.br. such as those of the genera Solanum and Capsicum $(2 \mathrm{n}=2 \mathrm{x}$ $=24)$ exhibit small chromosomes (1.2-2.4 $\mu \mathrm{m}$ for Solanum and 3.5-4.0 $\mu \mathrm{m}$ for Capsicum) and constancy in the distribution of meta- and submetacentric chromosomes (Bernardello and Anderson, 1990, Bernardello et al., 1994, Moscone, 1993). Nevertheless, the chromosomes of the Cestreae are the largest in the family, reaching up to $14 \mu \mathrm{m}$ and containing $1.36 \mathrm{pg}$ of DNA per chromosome (Berg and Greilhuber, 1992, 1993a,b, Sykorová et al., 2003a).

Berg and Greilhuber (1992, 1993a,b) studied five species of Cestrum ( $C$. aurantiacum, C. elegans, $C$. fasciculatum, C. parqui, and C. strigilatum) using several banding techniques, and found four different heterochromatic types: (i) large blocks unassociated with nucleolar organizing regions (NORs); (ii) numerous smaller blocks forming intercalary, proximal or distal dots; (iii) $\mathrm{CMA}_{3}{ }^{+}$ (chromomycin $\mathrm{A}_{3}$ ) bands associated with NORs; and (iv) DAPI $^{+}$(4'-6-Diamidino-2-phenylindole) bands associated with cold-sensitive regions (CSRs). The CSRs were first described by Dyer (1963) and at present have been reported in 11 genera of monocotyledons but only three dicotyledons. 
Members of Solanaceae show large diversity in heterochromatin accumulation and dispersion. Species of Capsicum exhibited heterochromatic blocks in all the centromeres and secondary constrictions, besides a variable number of large and small bands distributed along the chromosomes (Moscone et al., 1993). Similarly, Nicotiana species show localized DNA repetitive segments (microsatellites, ribosomal DNAs and telomeric sequences), while other (virus-associated) segments are dispersed along the chromosomes (Yoong Lim et al., 2000), and the literature suggests that Cestrum can also follows this model.

During the research reported in this paper we compared the karyotypes of four Brazilian Cestrum species $(C$. amictum (Schlecht.), $C$. intermedium (Sendtn.), $C$. sendtnerianum (Mart. \& Sendtn.) and C. strigilatum (Ruiz $\&$ Pav.)) using Feulgen staining and chromosomal markers produced by C-Giemsa and C-CMA 3 /DAPI banding, CSRs and fluorescence in situ hybridization (FISH) with $45 \mathrm{~S}$ and $5 \mathrm{~S}$ rDNA probes. The results are discussed considering the possible mechanisms of dispersion and distribution of heterochromatic segments and of rDNA cistrons in the karyotypes of the four species.

\section{Materials and Methods}

Seeds of the four Cestrum species cited above (eight populations) were obtained from different localities and cultivated in tubes in the seedling nursery of the Laboratory for Biodiversity and Restoration of Ecosystems (LABRE), Biology Science Centre, State University of Londrina, Paraná, Brazil. Vouchers were deposited at the FUEL herbarium (Table 1).

Slides were prepared of root tips pre-treated with $0.05 \%$ colchicine, fixed in Carnoy solution (ethanol/acetic acid 3:1, v:v), for up to $24 \mathrm{~h}$ and stored at $-20{ }^{\circ} \mathrm{C}$. Conventional staining was carried out by the Fuelgen method. The cover slips were removed after freezing slides in liquid nitrogen and the slides permanently mounted in Entellan. To obtain CSRs, roots were collected and pre-treated in Bristol nutritive solution (Bold, 1949) for $24 \mathrm{~h}$ at $0{ }^{\circ} \mathrm{C}$ and fixed in Carnoy solution. Roots were hydrolyzed in $1 \mathrm{M} \mathrm{HCl}$, dissected in a drop of $45 \%(\mathrm{v} / \mathrm{v})$ aqueous acetic acid and squashed. The cover slips were removed after freezing in liquid nitrogen and the preparations stained with 2\% (w/v) Giemsa and the cells photographed using Kodak Imagelink HQ ASA 25 film.

For chromosomal banding, roots were digested in an enzyme solution composed of $4 \%$ cellulase and $40 \%$ pectinase (both $\mathrm{w} / \mathrm{v}$ ) at $37^{\circ} \mathrm{C}$ and dissected in a drop of $45 \%$ acetic acid. After removal of the cover slips, the slides were aged for three days, and then incubated in $45 \%$ acetic acid, $5 \%$ barium hydroxide and $2 \mathrm{xSSC}, \mathrm{pH} 7,0$ (Schwarzacher et al., 1980, with modifications). The materials were stained in two different ways, either with $2 \%$ Giemsa or with the fluorochromes $0.5 \mathrm{mg} / \mathrm{mL} \mathrm{CMA}_{3}$ for $1.5 \mathrm{~h}$ and $2 \mu \mathrm{g} / \mathrm{mL}$ DAPI for $30 \mathrm{~min}$. Slides stained with Giemsa were mounted in Entellan and those stained with the fluorochromes mounted with a medium composed of glycerol/ McIlvaine buffer (pH 7.0) 1:1 plus $2.5 \mathrm{mM} \mathrm{MgCl}_{2}$. The cells were photographed using Kodak Imagelink HQ ASA 25 and T-max ASA 100 film.

Fluorescent in situ hybridization (FISH) was performed according to the procedures described by HeslopHarrison et al. (1991) and Cuadrado and Jouve (1994), with minor modifications. Slides were prepared as described for banding. The wheat probes $\mathrm{p} T a 71$ containing the $45 \mathrm{~S}$ rDNA sequence (Gerlach and Bedbrook 1979) and pTa794 containing the 5S rDNA sequence (Gerlach and Dyer 1980) were both labeled with biotin-14-dATP by nick translation and used for FISH. Each slide was treated with $30 \mu \mathrm{L}$ of hybridization mixture containing 100-150 ng of labeled probe $(4 \mu \mathrm{L}), 50 \%$ formamide $(15 \mu \mathrm{L}), 50 \%$ polyethylene glycol $(6 \mu \mathrm{L}), 20 \mathrm{xSSC}(3 \mu \mathrm{L}), 100 \mathrm{ng}$ of calf thymus DNA $(1 \mu \mathrm{L})$, and $10 \% \operatorname{SDS}(1 \mu \mathrm{L})$. The material was denatured at $90{ }^{\circ} \mathrm{C}$ for $10 \mathrm{~min}$, and hybridization was performed overnight at $37^{\circ} \mathrm{C}$ in a humidified chamber. Post-hybridization washes were carried out in $2 \times \mathrm{SSC}, 20 \%$ formamide in $0.1 \times \mathrm{SSC}$, $0.1 \times$ SSC and $4 \times \mathrm{xSC} / 0.2 \%$ Tween 20 , all at $42{ }^{\circ} \mathrm{C}$. The probes were detected with avidin-FITC conjugated and the post-detection baths were conducted in $4 \mathrm{xSSC} / 0.2 \%$ Tween 20 at room temperature. Slides were mounted in $26 \mu \mathrm{L}$ of a solution composed of antifade $(12.5 \mu \mathrm{L})$ and $50 \%$ glycerol in McIlvaine buffer (pH 7) with $2.5 \mathrm{mM}$

Table 1 - Chromosome measurements and morphology of the Cestrum species and populations analyzed. CL = Length of large and small chromosome pairs; THL $=$ Total length of haploid set.

\begin{tabular}{|c|c|c|c|c|c|c|c|}
\hline Cestrum species & Natural location in Brazil & $\mathrm{CL}(\mu \mathrm{m})$ & THL & Metacentric & Submetacentric & Acrocentric & FUEL \\
\hline C. amictum Schlecht. & Conquista farm, Rolândia, Paraná (PR) & 6.9 to 9.8 & 70.2 & 1.2.3.4. 6 and 7 & 5 & 8 & 30386 \\
\hline C. intermedium Sendtn. & $\begin{array}{l}\text { Mata dos Godoy State park, Londrina, PR } \\
\text { Mata Doralice reserve, Ibiporã, PR } \\
\text { Rubi valley, Londrina, PR }\end{array}$ & 7.3 to 11.8 & 77.3 & 1.2.3.5 and 7 & 4 and 6 & 8 & $\begin{array}{l}31197 \\
30255 \\
31398\end{array}$ \\
\hline C. sendtnerianum Mart. & $\begin{array}{l}\text { Hermógenes de Freitas Leitão Filho ecological } \\
\text { park, Campinas, SP }\end{array}$ & 6.4 to 8.7 & 63.4 & 1. 2.3 and 4 & 5. 6 and 7 & 8 & 33213 \\
\hline C. strigilatum Ruiz \& Pav & $\begin{array}{l}\text { São Jerônimo native Brazilian reserve, São } \\
\text { Jerônimo da Serra, PR } \\
\text { Sapopema, PR } \\
\text { Mata Doralice reserve, Ibiporã, PR }\end{array}$ & 7.2 to 8.6 & 63.3 & 1. 3. 4.6 and 7 & 2.5 and 8 & - & $\begin{array}{l}31953 \\
30984 \\
29682\end{array}$ \\
\hline
\end{tabular}


$\mathrm{MgCl}_{2}(12.5 \mu \mathrm{L})$, plus $1 \mu \mathrm{L}$ of $50 \mu \mathrm{g} / \mathrm{mL}$ propidium iodide. Photographs were taken using Kodak Proimage color ASA 100 film.

In general, 15 specimens were analyzed for each of the eight populations and idiograms constructed based on the analyses of about 10 metaphases and/or pre-metaphases obtained from each treatment. The chromosomes were classified based on the nomenclature proposed by Guerra (1986).

\section{Results}

All four Cestrum species studied had a diploid chromosome number of $2 \mathrm{n}=2 \mathrm{x}=16$, with chromosomes varying gradually in size from about $6.4 \mu \mathrm{m}$ to $11.8 \mu \mathrm{m}$ (Table 1 ), although $C$. intermedium exhibited slightly larger chromosomes. The predominant chromosome forms were meta- and submetacentric, but pair 8 of $C$. strigilatum was submetacentric while in the other species this pair was acrocentric (Table 1). Satellites were rarely seen, and the interphase nuclei were always reticulate. Karyotypical differences among the populations were not detected, even when the chromosomes were studied using several techniques (conventional staining, banding and FISH).

We found that C. amictum exhibited six metacentric pairs, one submetacentric and one acrocentric (Figure 1A, Table 1). C-Giemsa banding revealed heterochromatic blocks in all the chromosomes, with the intercalary bands being smaller and tenuous (or inconstant) in some preparations while the terminal bands were larger and more evident. The tenuous bands were distributed equidistantly on the long arms of almost all pairs except for pairs 2 and 8 . Large C-Giemsa blocks were visualized mainly in the terminal/subterminal regions of the short arms of the minor chromosome pairs (Figures 1E and 5A) but fluorochrome banding showed large $\mathrm{C}-\mathrm{CMA}_{3}{ }^{+}$blocks associated with NORs in the terminal regions of the short arms of pairs 6 and 8 , (Figures $2 \mathrm{~A}$ and $5 \mathrm{~A}$ ). The C-DAPI ${ }^{+}$bands were tenuous and slightly fluorescent, located in the terminal/subterminal regions of the short arms of pairs 6 and 7, and on the long arms of pairs 4 and 6 (Figures $2 \mathrm{~B}$ and 5A). Neutral bands $\left(\mathrm{C}-\mathrm{CMA}_{3}{ }^{0} \mathrm{DAPI}^{0}\right)$ appeared in the terminal regions of the short arm of pair 3 and proximal on the long arm of pair 8 (Figures 2A, 2B and 5A). Cold-sensitive regions (CSRs) were visualized in the intercalary region of the short arm of chromosome pair 4 of C. amictum (Figures $3 \mathrm{~A}$ and 5A). Terminal signals on the short arms of pairs 6 and 8 were detected with FISH with the $45 \mathrm{~S}$ rDNA probe, while FISH with the 5S rDNA probe gave a proximal signal on the long arm of pair 8 (Figures 4A, 4B and 5A).

The karyotype of $C$. intermedium was composed of five metacentric pairs, two submetacentrics and one acrocentric pair (Figure 1B and Table 1). After C-Giemsa banding, heterochromatic regions were visible in all the chromosomes (Figures 1F and 5B). Large C-Giemsa bands

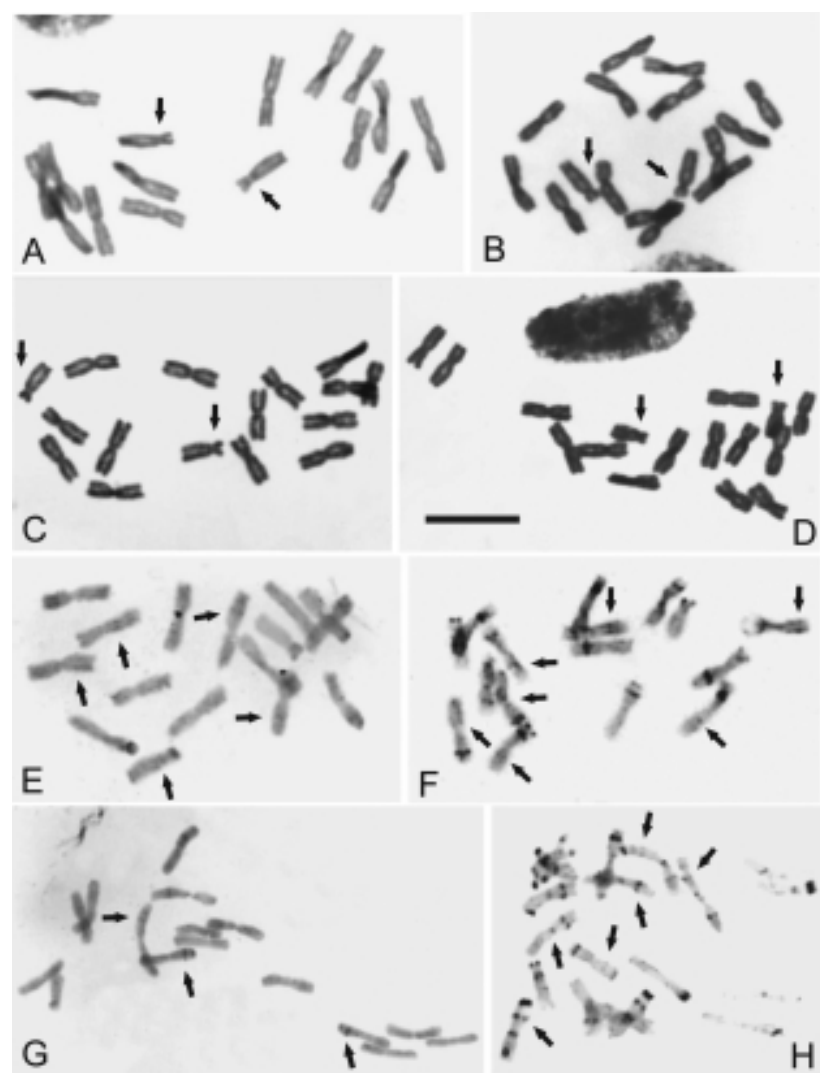

Figure 1 - (A-D) Feulgen-stained metaphases of Cestrum amictum (A), C. intermedium (B), C. sendtnerianum (C) and C. strigilatum (D). Arrows indicate the acrocentric pair. (E-H) Giemsa C-banded metaphases of Cestrum amictum $(\mathrm{E})$, C. intermedium $(\mathrm{F})$, C. sendtnerianum $(\mathrm{G})$ and $C$. strigilatum $(\mathrm{H})$. Arrows indicate some interstitial and inconspicuous $\mathrm{C}$ bands. Bar represents $10 \mu \mathrm{m}$.

were found in the intercalary/terminal positions of the long arms of pairs 4 and 5, in the terminal/subterminal regions of the short arm of pair 7 and 8 (both associated with NORs) and in the terminal regions of the short arms of pairs 2 and 3. More tenuous bands were detected at intercalary/terminal positions on the long arms of pairs 3 and 6, as well as in the intercalary part of the short arm of pair 6 . Weak and inconstant Pericentromeric C-Giemsa bands occurred in pairs 1, 2, 5, 6 and 7 (Figure 5B). Fluorochrome banding revealed $\mathrm{C}-\mathrm{CMA}_{3}{ }^{+} \mathrm{NOR}$-associated blocks adjacent to $\mathrm{C}$ $\mathrm{DAPI}^{+}$bands in the terminal and subterminal regions of the short arms of pairs 7 and 8 plus a tenuous neutral C$\mathrm{CMA}_{3}{ }^{0} / \mathrm{DAPI}^{0}$ band in the pericentromeric region of pair 8 . Weak $\mathrm{DAPI}^{+}$staining was also found in the pericentromeric region of pair 7 (Figures 2C, 2D and 5B). In some preparations cold-treatment (CSR) produced evidence of intercalary sites on the long arms of pairs 4 and 5 and C-Giemsa banding indicated that these sites were associated with large heterochromatic blocks (Figures 3B and 5B). Fluorescent in situ hybridization with the 45S rDNA probe revealed hybridization sites in the terminal region of the short arm of pair 7 and the subterminal region of pair 8 in addition to a weaker $\mathrm{C}-\mathrm{CMA}_{3}$ negative terminal site on 

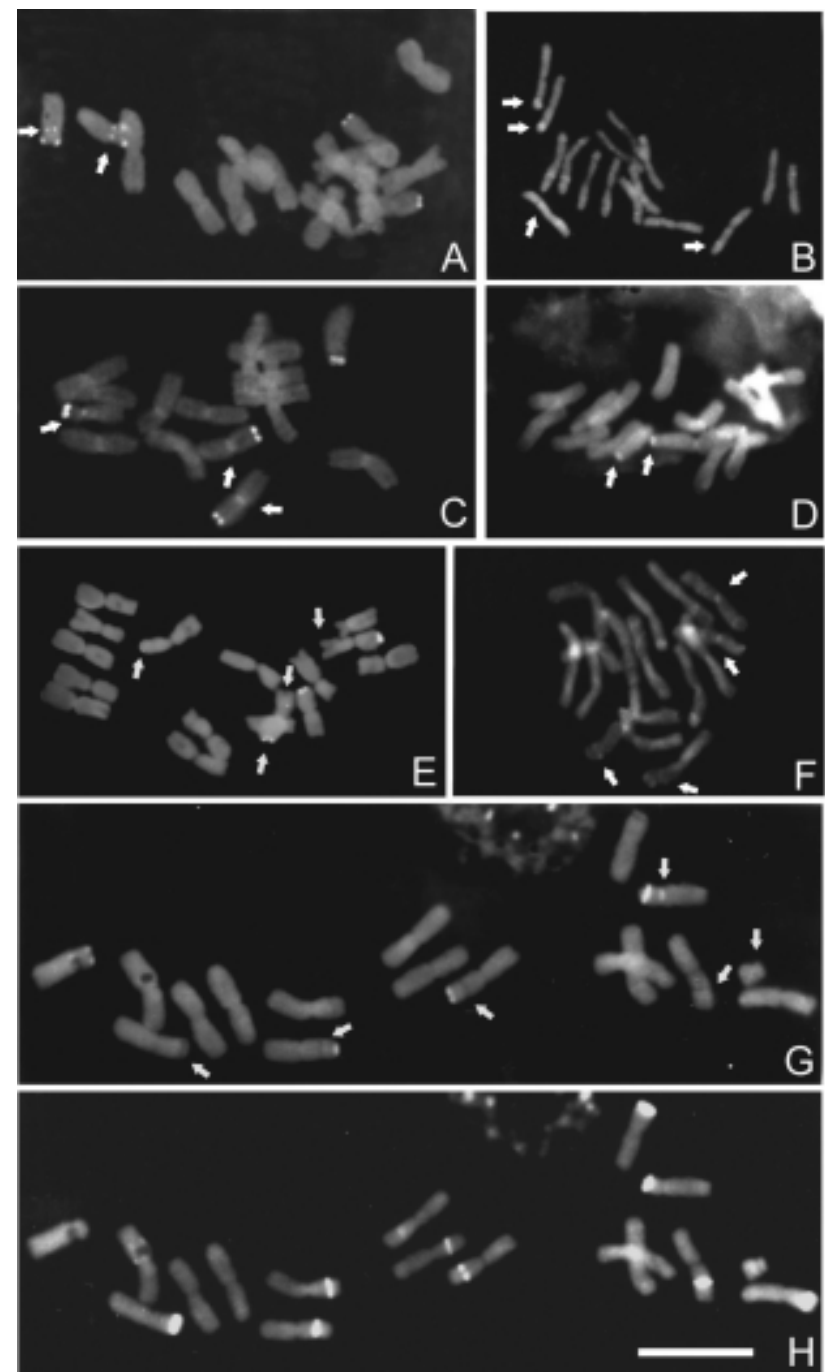

Figure 2 - Chromosome banding with fluorochromes in Cestrum species. (A) $\mathrm{C}-\mathrm{CMA}_{3}$ banding in $C$. amictum, arrows indicate the minor and proximal band in the pair 8. (B) C-DAPI banding in C. amictum, arrows indicate minor and interstitial bands. (C) C-CMA $\mathrm{C}_{3}$ banding in $C$. intermedium, arrows indicate inconstant centromeric-pericentromeric bands and minor and proximal band in the pair 8. (D) C-DAPI banding in C. intermedium, arrows indicate major terminal bands. (E). C-CMA $\mathrm{C}_{3}$ banding in $C$. sendtnerianum, arrows indicate minor and terminal bands. (F) C-DAPI banding in $C$. sendtnerianum, arrows indicate some proximal, terminal and interstitial bands. (G) $\mathrm{C}-\mathrm{CMA}_{3}$ banding in $C$. strigilatum, arrows indicate interstitial, proximal (pair 8) bands and a B-chromosome. $(\mathrm{H}) \mathrm{C}$ DAPI banding in C. strigilatum, arrows indicate minor interstitial bands and a B-chromosome. Bar represents $10 \mu \mathrm{m}$.

the long arm of pair 3. Hybridization sites with the $5 \mathrm{~S}$ rDNA probe were found near the centromere of the long arm of pair 8 (Figures 4D and 5B).

The karyotype of $C$. sendtnerianum consisted of four metacentric pairs, three submetacentrics and one acrocentric pair (Figure 1C and Table 1). After C-Giemsa banding, large blocks were observable in the terminal region of the short arm of pair 1 and in the proximal region of the short arm of pair 8. Large NOR-associated terminal blocks were revealed by $\mathrm{C}-\mathrm{CMA}_{3}$ banding in both arms of pair 3
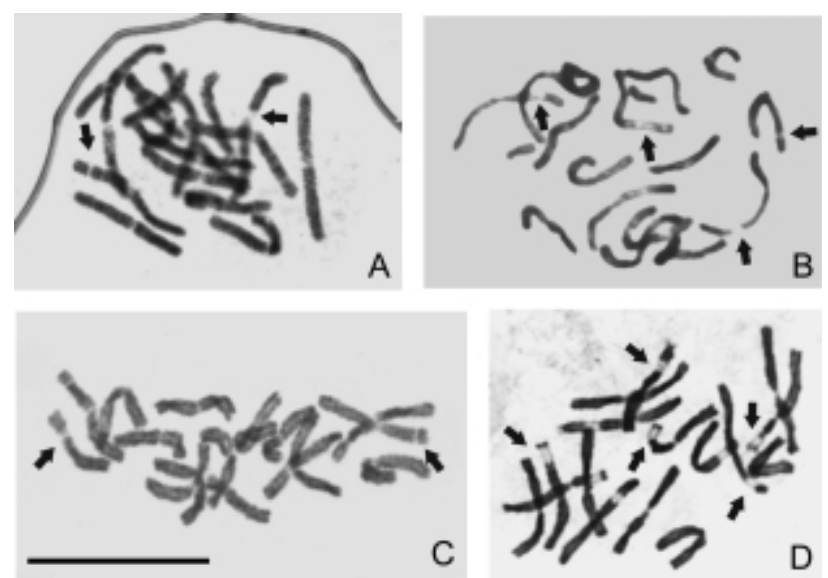

Figure 3 - Cold sensitive regions (CSRs) in Cestrum. (A) C. amictum, (B) C. intermedium, (C) C. sendtnerianum and (D) C. strigilatum. Arrows indicate terminal and interstitial CSRs. Bar represents $10 \mu \mathrm{m}$.

and on the short arm of pair 6. Some of these blocks also occurred in the intercalary regions of the short arms of pairs 1 , 2, 3 and 4, on the long arm of pair 6 , in the proximal region of the long arm of chromosome 7 and in both subterminal regions of pair 8. Equidistantly distributed tenuous and inconstant staining was found in the pericentromeric regions (mainly of the long arms) and intercalary/distal position of the long arms of all the chromosomes (Figure 1G). Fluorochrome banding showed NOR-associated terminal C$\mathrm{CMA}_{3}{ }^{+}$bands on both arms of pair 3 and the short arm of pair 6 , with another intercalary band (unassociated with NORs) also occurred on the short arm of pair 6. Tenuous $\mathrm{C}$-DAPI ${ }^{+}$bands were found at the subterminal regions of the short arms of pairs 3 and 7. One neutral (C$\mathrm{CMA}_{3}{ }^{0} / \mathrm{DAPI}^{0}$ ) band occurred in the pericentromeric region of pair 8 (Figures 2E, $2 \mathrm{~F}$ and $5 \mathrm{C}$ ). A single CSR was found at the intercalary position on the short arm of pair 7 , coinciding with a C-Giemsa/DAPI positive band (Figures 3C and 5C). Fluorescent in situ hybridization with the 45S rDNA probe revealed terminal signals in both arms of pair 3 , plus a terminal site on the short arm of pair 6 . In these three cases, the hybridization sites coincided with C-Giemsa and $\mathrm{C} / \mathrm{CMA}_{3}$ positive bands. A $\mathrm{CMA}_{3}$-negative $45 \mathrm{~S}$ rDNA hybridization site occurred in the terminal region of the long arm of pair 4. Hybridization with the 5S rDNA probe showed a proximal signal on the long arm of pair 8 (Figures 4C and 5C).

The karyotype of C. strigilatum is composed of five metacentric and three submetacentric pairs (Figure 1D and Table 1). After C-Giemsa banding, large intercalary blocks were observed on both arms of pair 1, on the long arms of pairs 3 and 7 and in the subterminal regions of the short arms of pairs 3 and 6 . Smaller blocks were visualized in the intercalary regions of both arms of pair 5, in the pericentromeric regions of pairs 5 and 6 and in the subterminal regions of pairs 6,7 and 8 . Except for pair 7, all the pairs showed equidistantly distributed tenuous or inconstant 

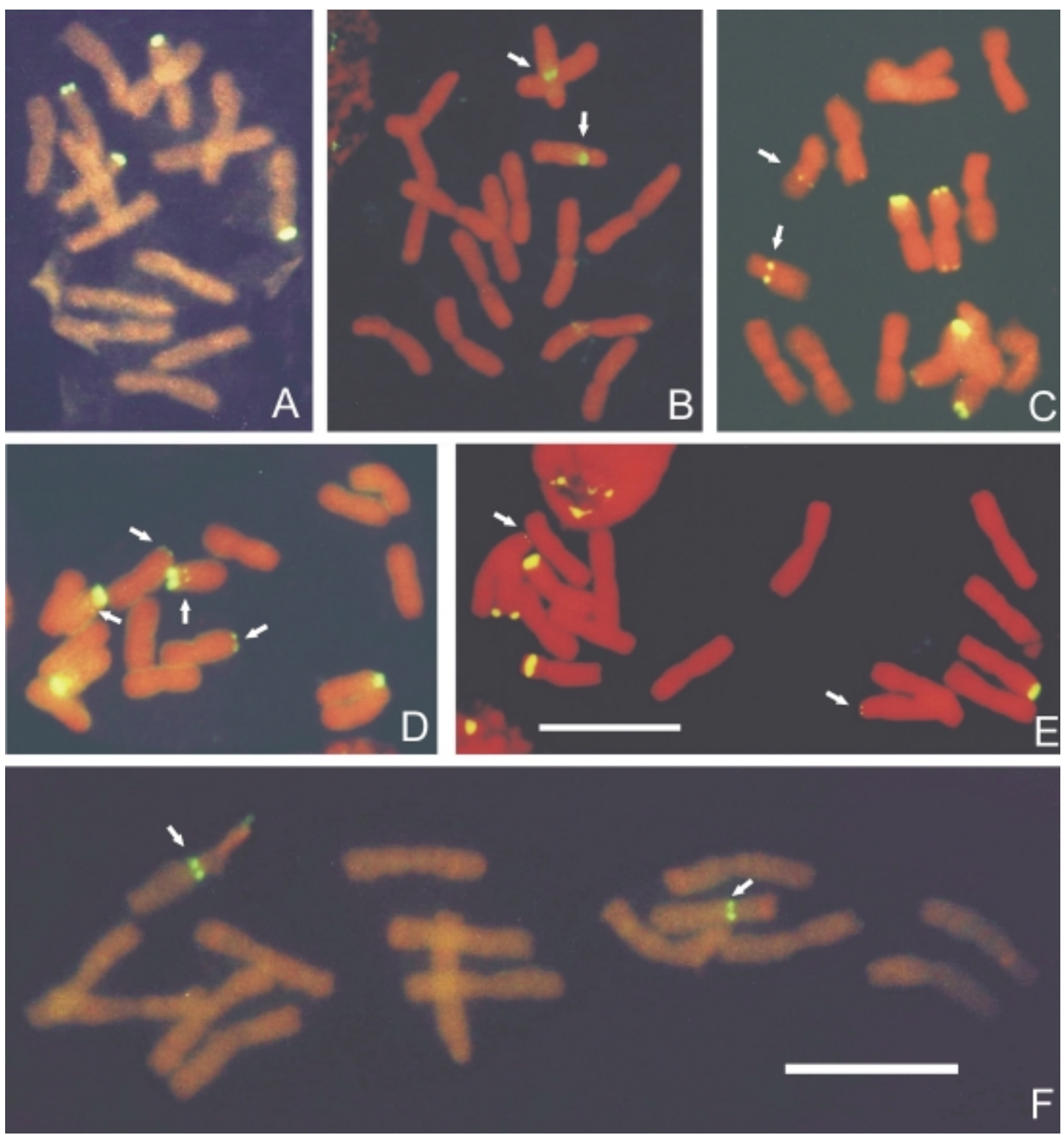

Figure 4 - Fluorescent in situ hybridization (FISH) with the pTa71 (45S rDNA) and pTa794 (5S rDNA) clones in Cestrum species. (A) Metaphase hybridized with $45 \mathrm{~S}$ rDNA probe in C. amictum. (B) Metaphase hybridized with 5S rDNA probe in C. amictum, arrows indicate out proximal signals. (C) Metaphase of $C$. sendtnerianum hybridized at the same time with $45 \mathrm{~S}$ and $5 \mathrm{~S}$ rDNA probes, arrows indicate terminal and tenuous $45 \mathrm{~S}$ rDNA signals (note a chromosome with two hybridization signals) and proximal 5S rDNA hybridization signals. (D) Metaphase of $C$. intermedium hybridized at the same time with $45 \mathrm{~S}$ and $5 \mathrm{~S}$ rDNA probes, arrows indicate terminal and tenuous $45 \mathrm{~S} \mathrm{rDNA}$ signals and proximal $5 \mathrm{~S}$ rDNA hybridization signals. (E) Metaphase of $C$. strigilatum hybridized with $45 \mathrm{~S}$ rDNA probe, arrows indicate a terminal and tenuous hybridization signal. (F) Metaphase of C. strigilatum hybridized with $5 \mathrm{~S}$ rDNA probe, arrows indicate proximal hybridization signals.

bands in their intercalary, proximal and distal regions (Figures $1 \mathrm{H}$ and $5 \mathrm{D})$. Distal $\mathrm{C}-\mathrm{CMA}_{3}{ }^{+}$bands, coinciding with NORs and C-Giemsa bands, were found on the short arms of pairs 7 and 8 (Figure 2G) and C-Giemsa associated C-DAPI ${ }^{+}$bands were intercalary on the short arms of pairs 2, 3, 6 and 7, subterminal in pairs 4 and 8 and the pericentromeric in pair 7 (Figure $2 \mathrm{H}$ ). A single neutral band was detected in the proximal region of the long arm of pair 8 (Figures 2G, 2H and 5D). Cold sensitive regions were consistently observed in association with the C-DAPI ${ }^{+}$ bands of pairs 4, 6, 7 and 8 , although the block observed on the short arm of pair 2 did not constantly exhibit a CSR and the C-DAPI ${ }^{+}$block in pair 3 was not induced by the cold (Figures 3D and 5D). We found that in situ hybridization with the $45 \mathrm{~S}$ rDNA probe gave a very similar distribution to that found in $C$. intermedium. There was a terminal signal on the short arm of pair 7 and subterminal in pair 8 and also a weaker $\mathrm{C}-\mathrm{CMA}_{3}$ negative terminal site on the long arm of pair 3. Fluorescent in situ hybridization with the 5S rDNA probe located a site next to the centromere on the long arm of pair 8 (Figures 4E, 4F and 5D). 


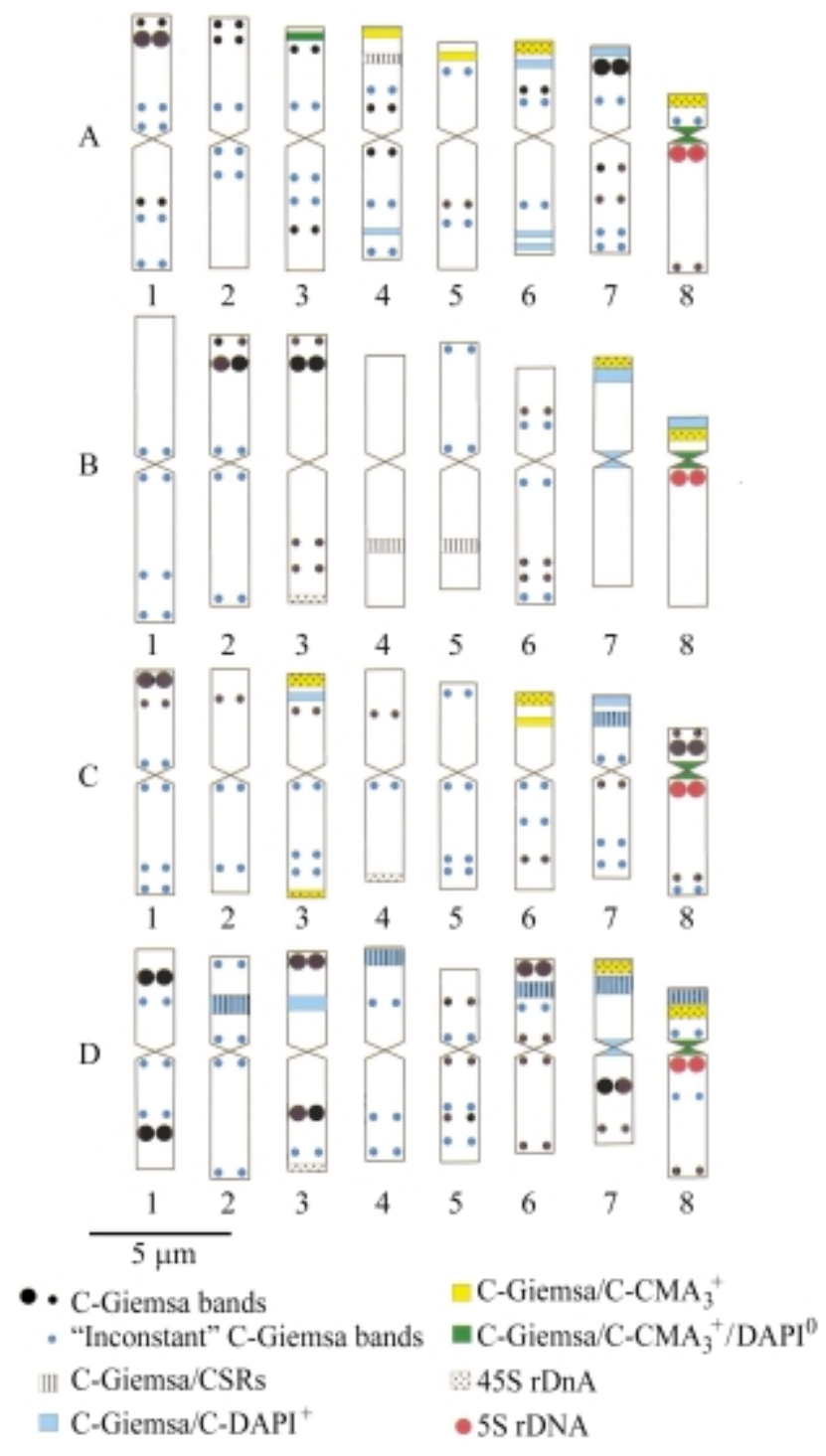

Figure 5 - Idiograms and chromosomal physical mapping of Cestrum amictum (A), C. intermedium (B), C. sendtnerianum (C) and C. strigilatum (D). The 'inconstant' bands correspond to small C-Giemsa ${ }^{+}$ bands that were not detected in all the preparation.

\section{Discussion}

The family Solanaceae shows karyotypical uniformity within the same genus mainly with relation to chromosome numbers and shapes. Illustrative examples are the species of the genera Solanum, with $2 \mathrm{n}=24$ (Bernardello and Anderson 1990, Bernardello et al. 1994), Lycium (Stiefkens and Bernardello 2002) and Capsicum (Moscone 1993). However, variation in the chromosome base number, with $\mathrm{x}=14$ and 17 , has also been reported for Hyoscyamus (Masoud et al. 1999). Species of Cestrum exhibit uniformity in chromosome number $(2 n=2 x=16)$ and type, including our new chromosome numbers for $C$. amictum and $C$. sendtnerianum. In general, the Cestrum karyotypes present meta- to submetacentric chromosome morphology for pairs 1 to 7 and subtelocentric for pair 8, as previously reported by Berg and Greilhuber (1992, 1993a,b). The Cestrum karyotypes accumulate numerical alterations related only to the occurrence of B chromosomes (Sykorová et al. 2003b, Fregonezi et al. 2004), except for a few structural changes probably associated with amplification and loss of repetitive DNA (Greilhuber 1992, 1993a,b).

The four Cestrum species studied by us showed heterochromatic blocks dispersed throughout all the chromosomes. The heterochromatic blocks varied in size, number, position and composition, this variation being most apparent when comparing the 14 large blocks present in 7 chromosome pairs of $C$. strigilatum with the 9 to 15 medium or large-sized blocks of $C$. intermedium, $C$. sendtnerianum and $C$. amictum. The same profile appeared when comparing the distribution of the smaller blocks and the inconstant dots within and between the species, these dots corresponding to very small C-Giemsa ${ }^{+}$bands that were not detected in all the preparations and were probably due to small variations in the banding and/or fixation conditions. The larger and smaller heterochromatic blocks preferentially occupied the intercalary and subterminal regions equidistantly. Five or more dots were found in some cases (e.g. C. amictum pairs 1, 2, 3, 4 and 7, $C$. intermedium pair 6 and $C$. strigilatum pair 5) while in the other chromosomes of the four species there were at most three or four dots. The preferential localization of particular heterochromatic segments in terminal and subterminal regions was also very evident for the C-CMA ${ }_{3}^{+}, \mathrm{C}-\mathrm{CMA}_{3}{ }^{+} / \mathrm{NOR}, \mathrm{C}-\mathrm{DAPI}^{+}$and $\mathrm{C}$-DAPI ${ }^{+} / \mathrm{CSR}$ bands, except for a few, most often intercalary, bands found in $C$. amictum (pair 4) and $C$. intermedium (pairs 6, 4 and 5), C. sendtnerianum (pairs 6 and 7) and C. strigilatum (pairs 2, 3, 6 and 7). Similar variation has been reported by Berg and Greilhuber (1992, $1993 a, b)$ in other five Cestrum species. According to Guerra (2000), quantitative and qualitative variation in heterochromatin could be common for different populations and species. Moscone et al. (1993) reported that the amount of heterochromatin in Capsicum varied by up to a factor of 10 , with about $4 \%$ of the total length of Capsicum chacoense chromosomes being occupied by C-bands and up to $33 \%$ of Capsicum campylopodium chromosomes containing mainly centromeric $\mathrm{C}$-bands along with a variable number of large and small distal bands and a few intercalary bands. Thus there are at least two possible heterochromatin dispersion modes in Cestrum species, non-equidistantly dispersion (Schweizer and Ehrendorfer 1976) in which isolated bands could have appeared from initial amplification sites and equidistant dispersion (Schweizer and Loidl 1987) in which a heterochromatic region could disperse repetitive segments to nearby heterologous chromosomal arms positioned at the same distance from the centromere in the 'chromosomal field' (Lima-de-Faria 1976). 
Berg and Greilhuber (1992, 1993a,b) found five different types of heterochromatin in Cestrum: (i) C-Giemsa ${ }^{+}$ bands, (ii) intercalary, proximal or distal C-Giemsa ${ }^{+}$dots, (iii) $\mathrm{CMA}_{3}{ }^{+}$bands associated with NORs, (iv) $\mathrm{CMA}_{3}{ }^{+}$ bands not associated with NORs, and (v) DAPI ${ }^{+}$bands associated with cold-sensitive regions (CSRs). Despite the different procedures employed by us our results confirm the existence of the same heterochromatic types plus two new types, i.e. $\mathrm{C}_{-} \mathrm{CMA}_{3}{ }^{0} / \mathrm{DAPI}^{0}$ neutral bands (see also Fregonezi et al. 2004) and C-Giemsa/CSR/DAPI negative bands.

We found that the number and distribution of the CSRs varied greatly between the Cestrum species studied, C. strigilatum showing a large number of CSRs while the other species showed few such sites. Variation in the size and number of CSRs has already been described in five other Cestrum species by Berg and Greilhuber (1992, 1993a, b), these authors having found a high number of CSRs in a different $C$. strigilatum population and a low number in $C$. parqui, even though the latter species has been considered the species with the larger amount of heterochromatin. In our study qualitative and quantitative variations were evident in both the heterochromatic segments and the CSRs, a fact to be expected because these regions consist of highly repetitive DNA segments. The occurrence of CSRs in terminal positions in C. aurantiacum (Berg and Greilhuber 1993b) and intercalary and subterminal positions in the four species studied by us also indicates that CSRs can be equidistantly dispersed, except for $C$. amictum and $C$. sendtnerianum because CSR segments appear in only one chromosome pair. In respect to AT and GC base composition, the CSRs were $\mathrm{CMA}^{-} / \mathrm{DAPI}^{+}$in $C$. strigilatum and $C$. sendtnerianum but negative for both fluorochromes in $C$. amictum and $C$. intermedium. In the species studied by Berg and Greilhuber (1992, 1993a,b), CSRs were always $\mathrm{CMA}^{-} / \mathrm{DAPI}^{+}$. Cold-sensitive $\mathrm{CMA}_{3}{ }^{+}$/ $\mathrm{DAPI}^{0}$ regions have been found in the genera Sambucus and Viburnum, Caprifoliaceae (Benko-Iseppon and Morawetz 1993). All these data suggest the existence of four CSR classes in plant genomes: (i) $\mathrm{C}-\mathrm{Giemsa}^{+} / \mathrm{DAPI}^{+}$, (ii) C-Giemsa ${ }^{+} / \mathrm{CMA}^{+}$, (iii) C-Giemsa ${ }^{+} / \mathrm{CMA}^{0} / \mathrm{DAPI}^{0}$ and (iv) $\mathrm{C}-G i e m s a^{+}$without fluorescence. However, the constitution of the CSR segments does not necessarily depend on the proportion of AT or GC bases but perhaps on the type of associated proteins or the different levels of methylation among the heterochromatic segments.

Conventional staining did not allow us to visualization secondary constrictions in any Cestrum chromosomes, difficulty in observing satellites or secondary constrictions having previously been described in Cestrum species by Berg and Greilhuber (1992, 1993a,b) using silver nitrate $\left(\mathrm{AgNO}_{3}\right)$ banding. In their study of a number of Cestrum species Berg and Greilhuber (1992, 1993a,b) found NOR-bearing areas in the terminal region of metacentric pair 1 and subtelocentric pair 8 of all the studied species ex- cept for Cestrum parqui, the NOR region of which was not in pair 8 but in two other metacentric pairs. We found that FISH with the $45 \mathrm{~S}$ and $5 \mathrm{~S}$ rDNA probes was useful in localizing rRNA genes in Cestrum. The occurrence of $45 \mathrm{~S}$ rDNA hybridization sites in the terminal regions of Cestrum chromosomes follows a common distribution pattern in the large majority of plant species (see Lima-de-Faria 1976). This profile has also been found in Capsicum (Moscone et al. 1995) and Nicotiana (Yoong Lim et al. 2000). In our study, $C$. amictum was the only species with four $45 \mathrm{~S}$ rDNA sites, supporting the initial proposal by Berg and Greilhuber (1992, 1993a,b). The other species showed more hybridization sites, three in $C$. intermedium and $C$. strigilatum and four in C. sendtnerianum. Hybridization also detected major and minor sites in $C$. strigilatum, $C$. sendtnerianum and $C$. intermedium, which could reflect intraspecific differences in the number of repeats. Although FISH is not a completely quantitative technique, the intensity of the signal can be related to the copy number of similar structures (Leicht and Heslop-Harrison 1992). We found that variation appeared not only in the number of rDNA sites but also in the organization of these segments, as seen in the occurrence of $45 \mathrm{~S}$ rDNA segments not associated with $\mathrm{C}-\mathrm{CMA}_{3}{ }^{+}$heterochromatin. We also found that in situ hybridization with the $5 \mathrm{~S}$ rDNA probe revealed a single proximal site on the long arm of pair 8 in the four species, also associated with a proximal Giemsa ${ }^{+} /$ $\mathrm{CMA}^{+} / \mathrm{DAPI}^{+}$band. In other genera such as Nicotiana (Yoong Lim et al. 2000, Kitamura et al. 2001) and Lycopersicon (Lapitan et al. 1991, Xu and Earle 1996) 5S rDNA sites are also located preferentially in the pericentromeric region of the long and short arms. The available information points to the conclusion that, at least for Cestrum species, the location of $45 \mathrm{~S}$ and $5 \mathrm{~S}$ rDNA sequences can be conserved in the chromosome field, in the terminal and subterminal region of some chromosomes $(45 \mathrm{~S})$ and next to the centromere on the long arm of pair $8(5 \mathrm{~S})$.

All the Cestrum species so far studied exhibited a uniform karyotype. We found that certain heterochromatic segments showed preferential localization (e.g. C-CMA ${ }_{3}^{+}$/ NOR blocks located terminally) or appeared in the same chromosome pairs in all species (e.g. C-Giemsa ${ }^{+} / \mathrm{CMA}^{0} /$ $\mathrm{DAPI}^{0}$ pericentromeric bands and $5 \mathrm{~S}$ rDNA sites in pair 8). However, the majority of heterochromatic segments showed variation in size and distribution among the species studied. This suggests that differentiation among Cestrum species occurred mainly through alterations in the organization of repetitive DNA segments, with few modifications in karyotype composition as observed by conventional staining.

\section{Acknowledgments}

The authors thank the Brazilian agencies Coordenação de Aperfeiçoamento de Pessoal de Nível Superior (CAPES) and Pró-Reitoria de Pesquisa e Pós-graduação 
(ProPPG-UEL) for financial support, Edson Mendes Francisco and Karina L. V. Ramalho de Sá for help with botanical material and Dr. Albert Leyva for help with the English.

\section{References}

Benko-Iseppon AM and Morawetz W (1993) Cold-induced chromosome regions and karyosystematics in Sambucus and $\mathrm{Vi-}$ burnum. Bot Acta 106:183-192.

Berg C and Greilhuber J (1992) Cold-sensitive chromosome regions and their relation to constitutive heterochromatin in Cestrum parqui (Solanaceae). Genome 35:921-930.

Berg C and Greilhuber J (1993a) Cold-sensitive chromosome regions and heterochromatin in Cestrum (Solanaceae): $C$. strigilatum, C. fasciculatum, and C.elegans. Plant Syst Evol 185:133-151.

Berg C and Greilhuber J (1993b) Cold-sensitive regions and heterochromatin in Cestrum aurantiacum (Solanaceae). Plant Syst Evol 185:259-273.

Bernardello LM and Anderson GJ (1990) Karyotypic studies in Solanum section Basarthrum (Solanaceae). Am J Bot 77:420-431

Bernardello LM, Heiser CB and Piazzano M (1994) Karyotypic studies in Solanum section Lasiocarpa (Solanaceae). Am J Bot 81:95-103.

Bold HC (1949) Bristol's solution and medium. http://www.pai. utexas.edu/research/utex/media/bristol.html. Accessed in 02/06/2003

Cronquist A (1988) The Evolution and Classification of Flowering Plants. The New York Botanical Garden, New York, $555 \mathrm{pp}$.

Cuadrado A and Jouve N (1994) Mapping and organization of highly-repeated DNA sequences by means of simultaneous and sequential FISH and C-banding in 6x-Triticale. Chrom Res 2:231-338.

D'Arcy WG (1986) Solanaceae: Biology and Systematics. Columbia University Press, New York, 603 pp.

Dyer AF (1963) Allocyclic segments of chromosomes and the structural heterozygosity that they reveal. Chromosoma 13:545-576.

Fregonezi JN, Rocha C, Torezan JMD and Vanzela ALL (2004) The occurrence of different Bs in Cestrum intermedium and C. strigilatum (Solanaceae) evidenced by chromosome banding. Cytogenet Genome Res 106:184-188.

Gerlach WL and Bedbrook JR (1979) Cloning characterization of ribosomal RNA genes from wheat and barley. Nucleic Acids Res 7:1869-1885.

Gerlach WL and Dyer TA (1980) Sequence organization of the repeting units in the nucleus of wheat which contain $5 \mathrm{~S}$ rDNA genes. Nucleic Acids Res 8:4851-4865.

Guerra M (1986) Reviewing the chromosome nomenclature of Levan et al. Rev Bras Genet 9:741-743.

Guerra M (2000) Patterns of heterochromatin distribution in plant chromosomes. Genet Mol Biol 23:1029-1041.

Heslop-Harrison JS, Schwarzacher T, Anamthawat-Jonsson K, Leitch AR, Shi M and Leitch IJ (1991) In situ hybridization with automated chromosome denaturation. Technique 3:106-109.

Kitamura S, Inoue M, Shikazono N and Tanaka A (2001) Relationship among Nicotiana species revealed by the 5S rDNA spacer sequence and fluorescence in situ hybridization. Theor Appl Genet 103:678-686.

Lapitan NLV, Ganal MW and Tanksley SD (1991) Organization of the 5S ribosomal RNA genes in the genome of tomato. Genome 34:509-514.

Leitch IJ and Hesolp-Harrison JS (1992) Physical mapping of the 18S-5.8S-26S rRNA genes in barley by in situ hybridization. Genome 35:1013-1018.

Lima-de Faria A (1976) The chromosome field I. Prediction of the location of ribosomal cistrons. Hereditas 83:1-22.

Masoud S, Maryam M and Mahboobeh K (1999) Karyological studies in Hyoscyamus species of Iran. Nord J Bot 19:369373.

Moscone EA (1993) Estudios cromosomicos en Capsicum (Solanaceae) II. Analisis cariotipico de C. parvifolium y $C$. annuum var. annuum. Kurtziana 22:9-18.

Moscone EA, Lambrou M, Hunziker T and Ehrendorfer F (1993) Giemsa C-banded karyotypes in Capsicum (Solanaceae). Plant Syst Evol 186:213-229.

Moscone EA, Loidil J, Ehrendorfer F and Hunziker AT (1995) Analysis of active nucleolus organizing regions in Capsicum (Solanaceae) by silver staining. Am J Bot 82:276-287.

Schwarzacher TP, Ambros P and Schweizer D (1980) Application of Giemsa banding to orchid karyotype analysis. Plant Syst Evol 134:293-297.

Schweizer D and Ehrendorfer FW (1976) Giemsa banded karyotypes, systematics, and evolution in Anacyclus (Asteraceae-Anthemideae). Plant Syst Evol 126:107-148.

Schweizer D and Loidl J (1987) A model for heterochromatin dispersion and the evolution of C-banded patterns. Chrom Today 9:61-74.

Stiefkens L and Bernardello G (2002) Karyotypic studies in Lycium section Mesocope (Solanaceae) from South America. Caryologia 55:199-206.

Sykorová E, Yoong Lim K, Chase MW, Knapp S, Leitch IJ, Leitch AR and Fakjus J (2003a) The absence of Arabidopsis-type telomeres in Cestrum and closely related genera Vestia and Sessea (Solanaceae): First evidence from eudicots. Plant J 34:283-291.

Sykorová E, Yoong Lim K, Fakjus J and Leitch AR (2003b) The signature of the Cestrum genome suggests an evolutionary response to the loss of (TTTAGGG) telomeres. Chromosoma 112:164-172.

Xu J and Earle ED (1996) Direct FISH of 5S rDNA on tomato pachytene chromosomes places the gene at the heterochromatic knob immediately adjacent to the centromere of chromosome 1. Genome 39:216-221.

Yoong Lim K, Matyasek R, Lichtenstein CP and Leitch AR (2000) Molecular cytogenetic analyses and phylogenetic studies in the Nicotiana section Tomentosae. Chromosoma 109:245-258.

Associate Editor: Marcelo Guerra 